\title{
Téoros
}

Revue de recherche en tourisme

\section{Le tourisme dans les parcs nationaux en Tunisie} Potentialités et contraintes

\section{Mohamed Souissi}

Volume 27, numéro 3, automne 2008

URI : https://id.erudit.org/iderudit/1070787ar

DOI : https://doi.org/10.7202/1070787ar

Aller au sommaire du numéro

Éditeur(s)

Université du Québec à Montréal

ISSN

0712-8657 (imprimé)

1923-2705 (numérique)

Découvrir la revue

Citer cet article

Souissi, M. (2008). Le tourisme dans les parcs nationaux en Tunisie :

potentialités et contraintes. Téoros, 27(3), 79-84.

https://doi.org/10.7202/1070787ar d'utilisation que vous pouvez consulter en ligne.

https://apropos.erudit.org/fr/usagers/politique-dutilisation/ 


\title{
Le tourisme dans les parcs nationaux en Tunisie
}

\author{
Potentialités et contraintes
}

\section{Mohamed Souissi}

Selon l'Organisation mondiale du tourisme (OMT), le tourisme basé sur la nature est "une forme de tourisme pour laquelle la découverte et l'observation de la nature sont la motivation et l'activité principale des clients". Cette définition assez globale et générale ne cache pas une certaine confusion terminologique lorsqu'on évoque l'activité touristique dans les parcs nationaux. Parle-t-on de tourisme vert, d'écotourisme ${ }^{1}$ ou de tourisme de randonnée? En effet, selon les responsables rencontrés à la Direction générale des forêts à Tunis, et en raison de la variabilité des champs d'activités, le tourisme dans les parcs nationaux englobe plus ou moins le tourisme vert, qui devrait inclure, en plus de l'observation de la nature, la visite des parcs et des sites culturels des espaces ruraux. Donc, on parlera du tourisme vert dans les parcs nationaux lorsque les randonnées et les visites dans les parcs constituent une composante importante de l'offre, en permettant au touriste une insertion dans le milieu naturel du parc visité ainsi que, dans la mesure du possible, une participation aux activités éducatives et scientifiques qui ont pour objectif la conservation du parc.

Malgré sa petite superficie par rapport à d'autres pays méditerranéens, la Tunisie dispose d'une diversité de paysages et de milieux naturels et d'un ensemble de parcs nationaux liés essentiellement à son type de climat et constituant une composante majeure des produits touristiques basés sur la nature ${ }^{2}$. En analysant la relation entre le tourisme et les parcs nationaux en Tunisie, l'objectif proposé ici est de voir comment on peut intégrer les parcs nationaux tunisiens dans le système touristique, tout en assurant leur fonction première qui est de protéger la Nature. Cette réflexion nécessite donc de s'interroger sur les potentialités et l'état des lieux du tourisme dans les parcs nationaux et d'analyser les contraintes que rencontre le développement d'un tourisme vert en Tunisie axé sur les parcs nationaux.

\section{Potentialités et état des lieux du tourisme dans les parcs nationaux tunisiens \\ Les parcs nationaux en Tunisie : un potentiel touristique}

La Tunisie est dotée d'un ensemble de parcs nationaux qui sont représentatifs des différents écosystèmes du pays et qui constituent par conséquent une composante majeure des produits touristiques basés sur la nature. Selon le code forestier de 1993, la Tunisie dispose de huit parcs nationaux qui s'étalent sur une superficie totale de 201752 hectares (tableau 1).

Les parcs nationaux tunisiens offrent des attraits touristiques, comme la possibilité de regarder les espèces dans leur habitat en parcourant celui-ci, d'organiser des circuits (safaris) photographiques ou d'autres activités analogues. Les différentes dimensions écologiques originales des parcs renferment en elles-mêmes un potentiel économique d'envergure : le tourisme basé sur la nature qui pourrait constituer un support de développement aussi bien pour les régions où sont localisés ces parcs que pour l'ensemble du pays. De même, certains parcs ne sont pas éloignés des aéroports internationaux et des grandes stations touristiques ${ }^{3}$, ce qui représente un potentiel complémentaire pour organiser des circuits et y attirer les touristes étrangers (ill. 1).
Selon notre enquête de terrain réalisée en 2003 auprès des responsables de la Direction générale des forêts à Tunis, quelques parcs nationaux en Tunisie disposent d'une infrastructure de base pour le tourisme vert. D'ailleurs, six parcs possèdent un écomusée, à savoir Ichkeul, Châambi, Bouhedma, Boukernine, Fîtja et Sidi Toui.

Le parc national d'lchkeul est l'un des plus importants parcs en Tunisie et dans le bassin méditerranéen. En plus de la diversité de ses biotopes (montagne, lac et marais), le parc bénéficie d'une richesse exceptionnelle en faune et en flore sauvages. II compte près de 230 espèces animales (180 espèces d'oiseaux, 21 de mammifères, 19 de reptiles et bactériens et 10 de poissons) et plus de 600 espèces végétales (Direction générale des forêts, 2003). Ce potentiel naturel et cette diversité ont fait que le parc national d'Ichkeul est reconnu «site du patrimoine mondial » sur la liste du patrimoine mondial naturel et culturel de l'Organisation des Nations Unies pour l'éducation, la science et la culture (UNESCO) et qu'il est inscrit sur la liste des zones humides d'importance internationale (Convention sur les zones humides signée à Ramsar en Iran en 1971) (Bouasker, 1995).

Le parc national d'lchkeul est considéré comme le parc le mieux équipé pour la pratique du tourisme de nature. Son écomusée, inauguré en 1989, dispose d'une exposition permanente de documentation scientifique permettant aux visiteurs de comprendre la richesse du parc et les enjeux de protection de l'environnement. De même, à partir de la plateforme de l'écomusée, des circuits touristiques longeant le flan du Djebel Ichkeul sont aménagés et proposent des aires de repos et des possibilités d'observation de la 


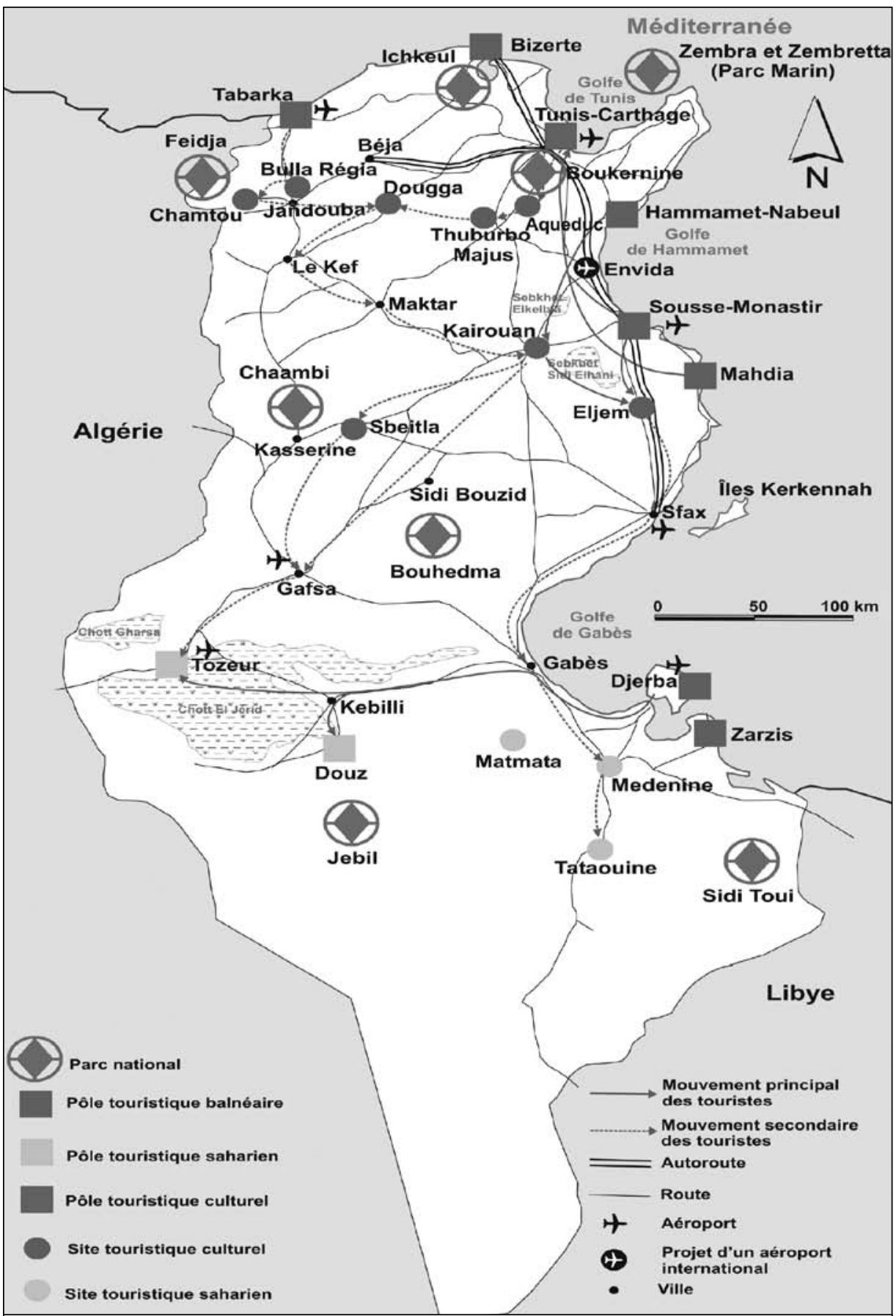

Illustration 1 : Les parcs nationaux et l'espace touristique en Tunisie.

Source : Enquêtes personnelles auprès de I'ONTT, la Direction générale des forêts, «Tunisie Voyages» à Tunis et « Nouvelles Frontières» à Paris (2003-2004) / Conception et réalisation personnelles.

faune et de la flore le long du parcours (Zaiane Ghalia, 2001). Enfin, l'emplacement du parc, à 25 kilomètres au sud-ouest du pôle touristique de Bizerte, est considéré comme un avantage en termes d'attraction touristique, notamment en raison des possibilités d'hébergement à Bizerte et de l'organisation de circuits pour la visite du parc.
En plus d'Ichkeul, il convient de noter que le parc national de Bouhedma, situé dans les régions de Gafsa et de Sidi Bouzid, abrite de son côté d'importantes richesses de la faune et de la flore (ill. 2) et qu'il est caractérisé par la présence de la savane subsaharienne dominée par le gommier (Acacia raddiana) ${ }^{4}$. Quelques animaux du parc-dont une grande partie réintroduite - sont exposés dans des enclos, les autres sont en liberté dans l'enceinte du parc. Son écomusée situé près du Bordj Bouhedma abrite un centre d'accueil, d'information et de recherche. À partir de l'emplacement de l'écomusée, une belle vue sur le Djebel Bouhedma et la forêt d'acacias s'offre au visiteur.

\section{Le tourisme dans les parcs nationaux : une activité touristique marginale}

Malgré l'importance des attraits touristiques des parcs nationaux tunisiens, il n'existe pas aujourd'hui une zone en particulier qui se distingue remarquablement, ou d'une renommée attirant des flux importants de touristes internationaux. Ces zones servent d'autres objectifs de préservation de l'environnement et de la biodiversité ainsi que des besoins récréatifs et de divertissement de la population nationale. Ils ne sont toutefois pas suffisamment valorisés; diverses possibilités de mise en valeur pour les loisirs et le tourisme vert sont encore ignorées. En général, les personnes fréquentant les parcs nationaux tunisiens sont très typiques. Les populations locales, fortement attachées à ces lieux, s'y rendent régulièrement, souvent en famille ${ }^{5}$; il s'agit aussi de jeunes écoliers lors de visites scolaires et de classes vertes (tableau 2). L'exemple d'Animaterre, un bureau tunisien spécialisé en matière d'environnement ainsi qu'en matière de conception et de développement de programmes éducatifs relatifs à ce domaine, est à encourager. Ce bureau propose aux institutions scolaires et aux amicales des entreprises d'organiser des semaines de découvertes et des sorties guidées dans les parcs nationaux (Zaiane Ghalia, 2002).

Les statistiques de la Direction générale des forêts (tableau 3) montrent clairement qu'lchkeul est, de loin, le parc national le plus visité. En 2002, le parc a accueilli 36280 visiteurs, dont près de $90 \%$ sont Tunisiens. Les nationalités étrangères les plus présentes sont les Français, les Allemands et les Britanniques, ce qui correspond parfaitement aux marchés les plus importants du tourisme tunisien. Le parc national de Sidi Toui accueille de plus en plus de visiteurs, du fait qu'il abrite le marabout de Sidi Bou Kthir, très vénéré par ses fidèles. Quant au nombre important de visiteurs tunisiens au 
Tableau 1

\begin{tabular}{|c|c|c|c|}
\hline \multicolumn{4}{|c|}{ Principaux éléments d'intérêt pour le tourisme vert dans les parcs nationaux tunisiens } \\
\hline $\begin{array}{l}\text { Parc national } \\
\text { Écosystème représenté }\end{array}$ & $\begin{array}{l}\text { Année } \\
\text { de } \\
\text { création }\end{array}$ & $\begin{array}{l}\text { Intérêts naturels } \\
\text { (flore, faune) }\end{array}$ & $\begin{array}{l}\text { Intérêts culturels } \\
\text { et historiques majeurs }\end{array}$ \\
\hline $\begin{array}{l}\text { Zembra et Zembretta } \\
5091 \text { ha } \\
\text { (zone insulaire méditerranéenne) }\end{array}$ & 1977 & $\begin{array}{l}\text { - Flore : } 266 \text { espèces. } \\
\text { - Flore marine : } 149 \text { espèces. } \\
\text { - Faune aquatique : } 200 \text { espèces et } 42 \text { espèces } \\
\text { de poissons. } \\
\text { - Avifaune : } 140 \text { espèces. }\end{array}$ & $\begin{array}{l}\text { - Présence de vestiges antiques. } \\
\text { - Ancienne occupation par une population } \\
\text { avec un caild. }\end{array}$ \\
\hline $\begin{array}{l}\text { lchkeul } \\
12600 \text { ha } \\
\text { (zone humide du nord) }\end{array}$ & 1980 & $\begin{array}{l}\text { - Flore : plus de } 600 \text { espèces } \\
\text { plus } 21 \text { espèces aquatiques. } \\
\text { - Avifaune : } 225 \text { espèces. }\end{array}$ & $\begin{array}{l}\text { - Présence de ruines antiques (habitations, } \\
\text { thermes, etc.). } \\
\text { - Présence de plusieurs marabouts. } \\
\text { - Systèmes de pêches antiques. } \\
\text { - Anciens bâtiments d'exploitation des sources. }\end{array}$ \\
\hline $\begin{array}{l}\text { Bouhedma } \\
16488 \text { ha } \\
\text { (Atlas saharien et steppe } \\
\text { subtropicale à acacias) }\end{array}$ & 1980 & $\begin{array}{l}\text { - Flore : environ } 400 \text { espèces. } \\
\text { - Faune et avifaune : non inventoriées. }\end{array}$ & $\begin{array}{l}\text { - Site préhistorique et lieux de culte néolithique. } \\
\text { - Marabouts, ruines romaines, tombes berbères, } \\
\text { anciennes mines. }\end{array}$ \\
\hline $\begin{array}{l}\text { Chaâmbi } \\
6723 \text { ha } \\
\text { (massifs de la Dorsale) }\end{array}$ & 1980 & $\begin{array}{l}\text { - Flore : environ } 262 \text { espèces, forêts de pins } \\
\text { d'Alep. } \\
\text { - Faune : pas de recensement précis. }\end{array}$ & $\begin{array}{l}\text { - Vestiges antiques importants non étudiés. } \\
\text { - Anciennes mines de plomb. }\end{array}$ \\
\hline $\begin{array}{l}\text { Boukornine } \\
1939 \text { ha } \\
\text { (collines du littoral nord-est) }\end{array}$ & 1987 & $\begin{array}{l}\text { - Flore : plus de } 600 \text { espèces. } \\
\text { - Faune : oiseaux ( } 50 \text { espèces), mammifères ( } 25 \\
\text { espèces) et papillons ( } 25 \text { espèces). }\end{array}$ & $\begin{array}{l}\text { - Présence de traces anciennes du temple de } \\
\text { Baal Saturne Balcarnansis et d'autres ruines } \\
\text { antiques. } \\
\text { - Tradition populaire de visite de la source Aîn } \\
\text { Zarga au printemps. }\end{array}$ \\
\hline $\begin{array}{l}\text { El Feidja } \\
2632 \text { ha } \\
\text { (environnement naturel de la } \\
\text { Kroumirie) }\end{array}$ & 1990 & $\begin{array}{l}\text { - Flore : } 700 \text { espèces, forêts de chênes zen et } \\
\text { de chênes liège. } \\
\text { - Faune : } 25 \text { espèces. } \\
\text { - Avifaune : } 70 \text { espèces. }\end{array}$ & $\begin{array}{l}\text { - Vestiges antiques importants non étudiés. } \\
\text { - Ruines de la guerre algérienne. }\end{array}$ \\
\hline $\begin{array}{l}\text { Jebil } \\
150000 \text { ha } \\
\text { (parcours saharien et grand erg } \\
\text { oriental) }\end{array}$ & 1995 & $\begin{array}{l}\text { - Flore et faune : non inventoriées. } \\
\text { - Site d'accueil de l'outarde houbara et de la } \\
\text { gazelle rym. }\end{array}$ & - Ancien campement berbère encore exploité. \\
\hline $\begin{array}{l}\text { Sidi Toui } \\
6315 \text { ha } \\
\text { (steppe du Sahara oriental) }\end{array}$ & 1998 & $\begin{array}{l}\text { - Flore et faune : non inventoriées. } \\
\text { - Site d'accueil de l'outarde houbara et de la } \\
\text { gazelle dorcas. }\end{array}$ & - Marabouts, ruines. \\
\hline
\end{tabular}

Sources : Direction générale des forêts (2003) et Zaiane Ghalia, 2002.

parc national de Boukornine, il s'explique, notamment, par la tradition populaire de visite de la source Aïn Zarga au printemps.

Selon les responsables de la Direction générale des forêts à Tunis, la plus grande affluence de visiteurs du parc national de l'lchkeul est enregistrée entre les mois de février et juin, soit presque cinq mois par an, alors que le reste de l'année les visites sont de moindre importance. En effet, d'octobre à janvier, il s'agit essentiellement de visiteurs intéressés par l'observation ornithologique, cette période correspondant à celle de migration des oiseaux. Au printemps, ce sont plutôt des visiteurs du grand public qui y viennent, à la recherche d'espace de détente pour des loisirs en plein air. En général, la priorité des visiteurs du parc de l'Ichkeul est accordée, essentiellement, à l'observation de la nature, en particulier la faune et la flore, et à la pratique d'une randonnée équestre ou pédestre à travers les différents sentiers du parc.

Ainsi, la tendance des entrées aux trois parcs nationaux les plus fréquentés (lchkeul, Boukornine, Sidi Toui) est le fruit de la combinaison de diverses données relatives aux conditions de ces parcs nationaux, par exemple l'accessibilité du site, la localisation du parc par rapport aux principales zones d'hébergements touristiques et les infrastructures d'accueil disponibles, particulièrement l'écomusée.
Les randonnées équestres ou à dos de dromadaire, la marche et le camping sont possibles dans beaucoup de parcs nationaux. Mais, à l'exception du parc Ichkeul, ces formes de tourisme sont faiblement pratiquées dans les autres parcs. De même, à part quelques groupes de touristes individuels, la plupart des agences de voyages qui organisent des randonnées touristiques dans les régions sahariennes n'incluent pas dans leur programme la visite des parcs. Selon notre enquête de terrain effectuée à Douz en avril 2004 et en mars 2005, la quasi-totalité des touristes interrogés (échantillon de 30 personnes) ignorent l'existence du parc national saharien de Jebil. Celui-ci est visité principalement lors 


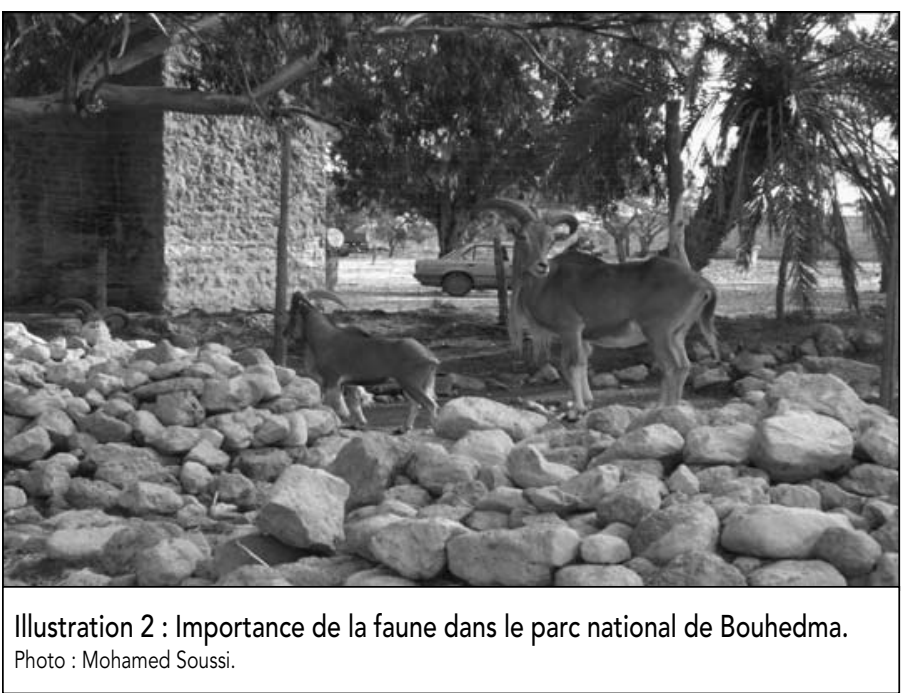

Tableau 2

\begin{tabular}{lcc}
\multicolumn{3}{c}{ Classification des visiteurs dans le parc national Ichkeul } \\
\hline Catégorie & $\mathbf{1 9 9 1}$ & $\mathbf{2 0 0 0}$ \\
\hline Élèves / Étudiants & $54,60 \%$ & $36,80 \%$ \\
Enseignants / Chercheurs & & $11,60 \%$ \\
Autres visiteurs & $45,40 \%$ & $51,60 \%$ \\
Total & $\mathbf{1 0 0} \%$ & $\mathbf{1 0 0 \%}$ \\
\hline Source : Zaiane Ghalia, 2002 : 219. & &
\end{tabular}

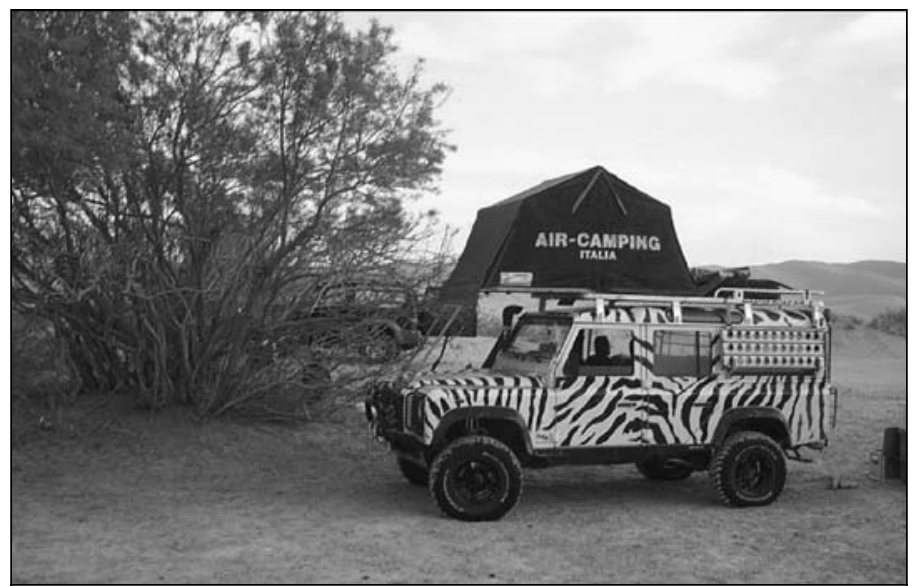

Illustration 3 : Touristes italiens en camping dans le parc national de Jebil. Photo : Mohamed Soussi.

\section{Tableau 3}

Effectifs de visiteurs tunisiens et étrangers dans les trois parcs nationaux tunisiens les plus fréquentés

\begin{tabular}{lccc} 
Parc national & Tunisiens & Étrangers & Total \\
\hline Ichkeul & 32430 & 3850 & 36280 \\
Boukornine & 17500 & 430 & 17930 \\
Sidi Toui & 16540 & 462 & 17002 \\
\hline Source : Direction générale des forêts, 2003. & &
\end{tabular}

d'excursions touristiques et scientifiques organisées pour des chercheurs étrangers et des amateurs de découverte de la biodiversité du Sud tunisien (ill. 3). Selon la Direction générale des forêts, il a été possible de relever, par exemple, qu'en 2003 les visiteurs de ce parc étaient 15 Tunisiens et 87 étrangers.

Enfin, il convient de noter que, malgré l'incitation du code forestier à la participation de la population locale aux actions d'aménagement, d'exploitation et de protection des parcs naturels, la question se pose sur les retombées économiques au profit de cette population. L'aménagement intérieur des parcs ne suffit pas, il faut aussi aménager les localités avoisinantes pour qu'elles puissent êtres intégrées dans les circuits touristiques des visites des parcs afin d'aboutir à une véritable intégration des populations locales. Ainsi, "le tourisme des parcs nationaux » est appelé au service du développement économique et social et de la conservation du patrimoine naturel.

\section{Contraintes et perspectives de développement touristique dans les parcs nationaux tunisiens \\ Les contraintes de l'intégration \\ des parcs nationaux dans le système touristique}

Les atouts dont disposent les parcs en Tunisie ne suffisent pas toujours à attirer les touristes. Malgré les efforts déployés ces dernières années et les programmes d'aménagement réalisés, ou en cours d'étude, les moyens financiers et logistiques manquent cruellement aux gestionnaires. Selon certains responsables de la Direction générale des forêts à Tunis, les fonds alloués annuellement à la gestion des parcs sont modestes et ne permettent pas la réalisation de projets de recherche importants ni d'aménagements adéquats. En effet, la majorité des réalisations qui ont vu le jour dans les parcs nationaux résultent de projets de coopération internationale. Par ailleurs, les parcs nationaux tunisiens ne sont pas systématiquement équipés d'un matériel puissant et conçu pour la pratique du tourisme vert (télescope, jumelle pour la vision nocturne...)

Pour le moment, les parcs nationaux ne sont pas suffisamment structurés pour répondre à une diversification du produit touristique. Ils ne disposent pas encore d'un véritable plan d'aménagement nécessaire à leur fonctionnement et à leur mise en valeur dans le cadre du développement du tourisme et des loisirs. Les parcs nationaux tunisiens sont encore principalement convoités par les populations locales pour les ressources naturelles (bois, zones de pâturage, etc.) qu'ils renferment et dont on les a privées par la création du parc. Cette absence de plan d'aménagement au niveau des parcs nationaux pourrait fort bien être le corollaire de l'absence de connaissances scientifiques approfondies relatives aux différents éléments naturels constituant ces parcs et de leur sensibilité. De même, entre zones écologiquement fragiles, zones sensibles et zones militaires, les autorisations nécessaires sont toujours multiples. Pour chaque visite de parc national, une autorisation spéciale doit être obtenue de la Direction 
générale des forêts. Les responsables de cette instance justifient ces contraintes administratives par la nécessité de sauvegarder le patrimoine naturel et d'en arriver à un développement durable (Souissi, 2007).

Au-delà des contraintes administratives, financières et logistiques, c'est le vide juridique dans lequel évolue le tourisme basé sur la nature qui pose problème. La réglementation du tourisme ne prend en considération que les activités se déroulant dans les zones définies comme touristiques ou sur les trajets d'hôtel à hôtel et les routes principales, voire vers des sites touristiques bien précis. De plus, même les parcs marins (îles de Zembra, Zembretta et la Galite), pour lesquels les amateurs de bateaux de plaisance et de plongée sous-marine montrent un intérêt particulier, ont un statut de parc ou de réserve. Ces endroits ne possèdent pas les structures d'accueil de base nécessaires pour accueillir quelques niches de touristes attirés par ces types de milieux naturels. En outre, l'accès à ces sites requiert l'obtention de diverses autorisations auprès des administrations concernées (Direction générale des forêts, ministère de la Défense, Marine marchande, Police maritime, Capitainerie et Office du tourisme). Actuellement, ces îles attirent de 400 à 600 visiteurs par année, essentiellement des amateurs de la nature, des chercheurs et des scientifiques tunisiens et étrangers (Direction générale des forêts, 2003).

Les parcs nationaux en Tunisie doivent par ailleurs prendre en compte tant les impératifs du développement économique - basé sur l'utilisation des ressources naturelles - que les exigences de protection et de sauvegarde des dimensions écologiques de ces parcs. En effet, au cours de notre enquête de terrain, certains ingénieurs des forêts précisent qu'il existe dans quelques régions des objectifs opposés entre les gestionnaires des parcs et les acteurs locaux et régionaux. Alors que les gestionnaires tentent de conserver au mieux le patrimoine naturel et culturel des parcs, les acteurs locaux et régionaux, qu'ils soient publics ou privés, cherchent le plus souvent à développer une activité touristique forte afin de faire bénéficier les populations locales des retombées économiques éventuelles liées à l'installation d'une telle structure.
De leur côté, les responsables de la Direction générale des forêts affirment qu'ils ne cherchent pas à renforcer la présence touristique dans les parcs naturels, s'appuyant justement sur la priorité de préserver et de valoriser leur aspect environnemental. Toutefois, ils ne sont pas contre le développement d'une activité touristique "douce" et contrôlée qui s'engage à respecter quelques conditions imposées par la Direction (nombre limité de visiteurs, suivi des circuits touristiques autorisés, encadrement des visites par guide connaisseur du parc...).

De ce fait, si la pression touristique est trop pesante, le milieu sera dégradé et les touristes seront insatisfaits, ces derniers recherchant en effet des lieux plutôt beaux et sauvages offrant bien sûr une certaine logistique d'accueil. Les touristes s'attendent donc à ce que le parc ne soit pas surpeuplé et ils exigent une certaine qualité sur les plans de l'infrastructure, du paysage, de la propreté et des espèces naturelles phares (Marchand, 2003). Enfin, la gestion et la planification des parcs nationaux restent à organiser; ils pourraient bénéficier de l'expérience de certains autres dans le monde (Espagne, France, Canada...) afin d'éviter des pièges liés essentiellement à un développement incontrôlé du tourisme qui pourrait entraîner des effets néfastes sur la faune et la flore de ces parcs naturels.

\section{L'avenir du tourisme dans les parcs nationaux : proposition de nouvelles pistes}

En tenant compte du potentiel naturel des parcs nationaux tunisiens, plusieurs recommandations peuvent être faites pour l'intégration des parcs nationaux dans le réseau touristique :

- Inciter les opérateurs tunisiens qui se spécialisent dans le tourisme vert à s'impliquer dans l'élaboration de produits répondant le mieux possible à la fois aux attentes des touristes et aux besoins des populations locales et faciliter cet engagement sous toutes les formes possibles, notamment par des privilèges fiscaux et des textes relatifs à l'accès aux parcs nationaux.
- Encourager les pouvoirs publics et les acteurs privés à renforcer les actions «environnement» du parc. En effet, sans un environnement exemplaire, que ce soit en matière de paysages ou de qualité du patrimoine bâti, il est illusoire de vouloir attirer les touristes pour des séjours prolongés et donc de mettre en place une réelle politique de produit touristique. En France, par exemple, la véritable randonnée a été particulièrement favorisée par la création des parcs nationaux et des parcs naturels régionaux, ainsi que par le développement d'une politique de création d'itinéraires sur l'ensemble du territoire, en concertation avec l'État, les collectivités locales et le Comité national des sentiers de grande randonnée (Michaud, 1983).

- Mettre en place de véritables produits pour afficher une meilleure offre touristique des parcs. Les produits "nature» doivent être des séjours qui permettent de découvrir les parcs, leurs richesses naturelles et patrimoniales. Mais nous savons que ces richesses, si intéressantes soient-elles, ne suffisent pas si elles ne sont pas mises en valeur par une chaîne de prestations de qualité homogène, présentant une constance et une fiabilité irréprochable (Boo, 1991).

- Identifier d'autres acteurs - en dehors des professionnels du tourisme - pouvant gérer les activités touristiques de loisirs et de randonnées dans les forêts et les parcs nationaux (organisations non gouvernementales [ONG], associations de protection de la nature, associations de randonnées équestres et pédestres, populations locales...), en collaboration avec la Direction générale des forêts et l'Office du tourisme tunisien (ONTT). De même, il faut favoriser la collaboration entre les agences de voyages spécialisées en tourisme vert et les ONG locales. Celles-ci sont en contact avec les populations et constituent des intermédiaires efficaces pour les agences de voyages, notamment pour l'identification de produits locaux et de personnes pouvant intervenir dans le tourisme orienté vers la nature. Elles peuvent également faciliter le dialogue entre les voyagistes et les populations locales. 
- Mettre en place des infrastructures d'accueil et d'hébergement (petit hôtel adapté à caractère familial, gîte rural, espace de camping...). Selon les entretiens que nous avons menés avec les responsables de la Direction générale des forêts à Tunis, la Tunisie dispose d'un nombre important d'anciennes structures légères destinées au tourisme vert qui peuvent être réhabilitées pour héberger les touristes de randonnée. C'est le cas des maisons forestières ou des fermes coloniales dans les régions d'Elkef, de Béja et de Tabarka, dans le nord-ouest tunisien (Souissi, 2007).

- Aménager des circuits pour les randonnées et les promenades pédestres en tenant compte des principes de conservation du milieu naturel, afin d'éviter que les visiteurs s'aventurent n'importe où et n'importe comment dans le parc (Zaiane Ghalia, 2002). L'aménagement d'une variété de sentiers de promenades thématiques permettrait l'instauration de "circuits à la carte". Le visiteur pourra alors, selon la distance à parcourir, le temps nécessaire et les sujets proposés, choisir le parcours qu'il effectuera durant sa visite. Par conséquent, il est nécessaire de procéder à une étude complète de chaque circuit proposé, de façon à pouvoir indiquer au visiteur non seulement ce qu'il pourra y découvrir, mais aussi la distance qu'il aura à parcourir, les difficultés potentielles qu'il pourrait rencontrer et le temps moyen qu'il lui faudra pour compléter la promenade.

Les parcs nationaux tunisiens pourront développer dans l'avenir un produit touristique dynamique, s'appuyant sur la base même de leur existence, à savoir la protection et la mise en valeur de leurs richesses naturelles et patrimoniales. Cependant, les pouvoirs publics doivent soutenir toutes les initiatives émanant des entreprises du secteur privé, en adéquation avec l'émergence de nouveaux besoins touristiques à satisfaire et contribuant à développer, de façon directe ou indirecte, le tourisme vert en Tunisie, à l'exemple d'autres pays méditerranéens (l’Espagne, la France...).

\section{Conclusion}

Ce travail a permis de montrer les potentialités, la réalité, les contraintes et les perspectives du développement du tourisme dans les parcs nationaux tunisiens, constituant l'essentiel de ce qu'on appelle généralement le tourisme vert, le tourisme doux ou alternatif. Cependant, il paraît utile aujourd'hui de rappeler que la connaissance sur ces formes de tourisme et sur leurs effets induits est très embryonnaire. II est difficile de connaître avec précision les retombées économiques et sociales de ces types d'activités. Mais, pour le système touristique tunisien, ces pratiques peuvent redéfinir un tourisme plus diversifié et plus équilibré dans l'espace et dans le temps, valorisant davantage les richesses naturelles, mais aussi patrimoniales, historiques et socioculturelles. Ce potentiel devient de plus en plus important, surtout lorsqu'on connaît certaines difficultés du tourisme de masse : aujourd'hui les voyagistes sont souvent incapables de satisfaire la clientèle qui est avide de rencontrer la nature, les cultures etc. II s'agit d'un véritable avantage pour le tourisme vert dans les parcs nationaux, à condition de créer une offre structurée de «produits verts» qui réponde aux exigences de cette clientèle. Dans l'avenir, le tourisme dans les parcs nationaux pourrait jouer le même rôle que le tourisme saharien qui a apporté une dimension complémentaire au tourisme balnéaire, notamment par le biais de randonnées de découverte des parcs dans leurs milieux naturels.

Mohamed Souissi est enseignant chercheur au Département de géographie à la Faculté des lettres et des sciences humaines de Sousse, Tunisie.

\section{Notes}

1 En général, le terme écotourisme correspond à un tourisme respectueux de l'environnement et des communautés locales (selon l'OMT).

2 Le parc regroupe un ou plusieurs écosystèmes et a une mission bien déterminée : la conservation, l'éducation, l'utilisation rationnelle des ressources naturelles, la recherche scientifique et la récréation. Dans un parc, l'intervention humaine est tolérée et des plans de gestion sont élaborés (selon le code forestier de la république tunisienne, 1993).

3 Par exemple, Ichkeul est situé à 75 kilomètres au nord de la capitale Tunis et à 25 kilomètres au sud-ouest de Bizerte. De son côté, le parc de Boukernine n'est qu'à 18 kilomètres au sud de Tunis.

4 Acacia raddiana ou Acacia tortilis, espèce végétale rare appartenant à la savane subsaharienne.

5 Par exemple, la majorité des visiteurs du parc national Ichkeul sont originaires des régions de Tunis, de Bizerte, de Menzel Bourguiba et de Mateur.

\section{Bibliographie}

Bouasker, R. (1995), «Le parc national de l'Ichkeul », Les zones protégées en Méditerranée : espaces, espèces et instruments d'application des conventions et protocoles de la Méditerranée, publication Centre d'études, de recherches et de publication, Tunis, p. $79-88$.

Boo, E. (1991), "Faire de l'écotourisme une entreprise durable : recommandation concernant son organisation, son développement et sa gestion ", dans T. Whelan (dir.), L'écotourisme : gérer l'environnement, Paris, Nouveaux horizons, p. 179-190.

Direction générale des forêts (2003), La flore et la faune dans les parcs nationaux tunisiens, rapport général, Tunis.

Direction générale des forêts (2003), Statistiques des parcs nationaux tunisiens, rapport annuel, Tunis.

Marchand, H. (2003), Les parcs nationaux marins, insulaires et littoraux : essai autour d'un modèle spatial de gestion des grands espaces naturels mondiaux, thèse de doctorat en géographie, Paris, Université de Paris IV-Sorbonne.

Michaud, J.L. (1983), Le tourisme face à l'environnement, Paris, Presses universitaires de France, coll. "Le géographe».

ONTT (Office national du tourisme tunisien) et Agence japonaise de coopération internationale (2001), Étude du plan directeur de développement du tourisme en République tunisienne, esquisse du rapport final, tome III, Tunis.

ONTT (Office national du tourisme tunisien) et Agence japonaise de coopération internationale (2001), Etude du plan directeur de développement du tourisme en République tunisienne, rapport d'avancement, Tunis.

Souissi, M. (2007), Le tourisme international en Tunisie : vers de nouvelles formes et la réorganisation de l'espace touristique, thèse de doctorat en géographie, Paris, Université de Paris IV-Sorbonne.

Zaiane Ghalia, S. (2001), "Tourisme écologique et culturel dans les parcs nationaux tunisiens et intégration des populations locales", dans N. Sekik (dir.), Patrimoine et co-développement durable en Méditerranée occidentale, actes du séminaire international, Tunis, UNESCO, p. 295-307.

Zaiane Ghalia, S. (2002), Tourisme et loisirs dans les parcs nationaux tunisiens : l'exemple du parc national de l'Ichkeul, thèse de doctorat en géographie, Aix-en-Provence, Université de Provence. 